\title{
Construction of an Efficacious Model for a Nondestructive Identification of Traditional Chinese Medicines Liuwei Dihuang Pills from Different Manufacturers Using Near-infrared Spectroscopy and Moving Window Partial Least-squares Discriminant Analysis
}

\author{
Hai-Yan Fu, Shuang-Yan Huan, ${ }^{\dagger}$ Lu Xu, Jian-Hui Jiang, Hai-Long Wu, ${ }^{\dagger}$ Guo-Li ShEn, and \\ Ru-Qin Yu
}

State Key Laboratory of Chemo/Biosensing and Chemometrics, College of Chemistry and Chemical Engineering, Hunan University, Changsha 410082, China

\begin{abstract}
This paper reports on the construction of an efficacious model for a non-invasive identification of traditional Chinese medicines, Liuwei Dihuang pills from different manufacturers, on the basis of near-infrared spectra (NIRS) coupled with moving window partial least-squares discriminant analysis (MWPLSDA). Considering the continuity of near-infrared spectral measurements, MWPLSDA is used to identify continuous and highly classification-related information intervals, a simple, yet effective classification model that can be developed for identifying accurate 150 Liuwei Dihuang pills from five different manufacturers. Meanwhile, the method is compared with some traditional pattern-recognition methods including principal component analysis (PCA), linear discriminant analysis (LDA) and partial least-squares discriminant analysis (PLSDA). The obtained results show that the method not only can reduce the operation time, but also significantly improves the classification accuracy. Hence, the nondestructive method can be expected to be promising for more practical applications on quality control and the discrimination of traditional Chinese medicine.
\end{abstract}

(Received November 18, 2008; Accepted February 3, 2009; Published September 10, 2009)

\section{Introduction}

In traditional Chinese medicine, Liuwei Dihuang pills (Pills of Six Ingredients with Rehmanniae) is prepared by six kinds of herbs, including Radix Rehmanniae Preparata (Shu Di Huang), Rhizoma Dioscorcae (Shan Yao), Fructus Corni (Shan Zhu Yu), Rhizoma Alismatis (Ze Xie), Poriae (Fu Ling) and Cortex Moutan Radicis (Mu Dan Pi). Modern pharmacological research has shown that it is not only helpful for curing irritability coryza, chloasma and diabetes, but also as an advantage to regulating the immune function and preventing oesophagus cancer. Based on its broad use, there are many manufactures in the industry of producing this kind of medicine. Different manufacturers can produce the same products using the same formula or formula with minor differences. The quality of the medicinal herbs and the producing techniques would largely influence the efficacy of the final products. Worse still, some manufacturers' production of Liuwei Dihuang is not according to the standard regulations, which results in an inferior quality of the finished product, and the substandard Liuwei Dihuang pills may do harm to the intestine and stomach of humans. Thus, the quality of Liuwei Dihuang pills should be controlled urgently. It is therefore valuable to develop an applied method to discriminate Liuwei Dihuang pills from different manufacturers with very much the

$\dagger$ To whom correspondence should be addressed. E-mail: syhuan@hnu.cn (S.-Y. H.); hlwu@hnu.cn (H.-L.W.) same formula. This will help promote intrinsic quality control of the traditional Chinese medicine, prevent infringements and also beat fake and shoddy products in the open market.

The basic formula of Liuwei Dihuang pills is as follows: $32 \%$ Radix Rehmanniae Preparata, 16\% Rhizoma Dioscorcae, 16\% Fructus Corni, 12\% Rhizoma Alismatis, 12\% Poriae and 12\% Cortex Moutan Radicis. According to the China Pharmacopoeia ${ }^{1}$ for rough medicines, the content of paeonol must be no less than $1 \mathrm{mg}$ and $0.7 \mathrm{mg}$ per gram for Shui Mi Wan and Xiao Mi Wan, respectively; and the content of ursolic acid of Fructus Corni must be more than $0.2 \mathrm{mg}$ and $0.13 \mathrm{mg}$ for Shui Mi Wan and Xiao Mi Wan, respectively. Also the content of stone of Fructus Corni must be lower than 3\%. Thus, these ingredient contents are always used as a standard to control the quality of Liuwei Dihuang pills. However, according to an investigation on the Liuwei Dihuang pills in the market place, the content of stone of Fructus Corni is seldom in accord with the standard; in some cases it even soars to $30 \% .^{2}$ The medicines were adulterated, which inevitably resulted in a reduction of the effective ingredients and the contents. The previous idetification methods were always based on the contents of one or more principal components in the herb. Unfortunately, theses methods involve a large number of tedious analytical tasks, such as chromatography. Before injection of the extracts into the chromatography column, the samples must be carefully washed, dried and extracted. Further, the samples are multiple component systems with a synergistic effect. Therefore, one or more principal components could neither characterize the samples 
well, nor be used as a precise criterion for the identification and quality-control of traditional Chinese medicine.

In recent years, the rapid development of near-infrared spectroscopy (NIRS) ${ }^{3,4}$ as an effective, simple, non-destructive and green method for the "fingerprint" characterization of the herb samples including complex components might light a new avenue to simple experimentation, which is introduced and applied necessarily in quality identification analysis of Chinese medicine. However, because of the severe overlap of the absorption bands in NIRS, it is difficult to interpret the NIR spectra directly. Hence, in order to achieve excellent prediction accuracy of quality classification, chemometrics methods of preprocessing spectral signal, extracting the characteristic information on the chemical composition and developing robust classification models must be provided.

In the present work, moving window partial least-squares (MWPLS) was used as an interval selection method and moving window partial least-squares discriminant analysis (MWPLSDA) for the identification of the traditional Chinese medicines Liuwei Duang pills from different manufacturers is proposed. Although it is the first time to be utilized in the discriminant analysis of the traditional Chinese medicines, Liuwei Duang pills from different manufacturers, the strategy has been successfully employed for the identification of different kinds of bezoar. MWPLS cons of spectral variables and selects intervals in terms of less complexity and lower calibration errors. A salient advantage of MWPLS is that the calibration model is very stable against interference from non-composition-related factors. In studies reported by Ozaki et al., ${ }^{6-13}$ MWPLS was successfully applied to spectral interval selection for calibration problems, and obtained desirable results. MWPLSDA can also benefit from less complexity and lower training errors for classification problems of spectral data with continuous variables. Compared to traditional pattern-recognition methods, including principal component analysis (PCA), linear discriminant analysis (LDA), partial least-squares discriminant analysis (PLSDA) for the classification analysis, the obtained results reveal that MWPLSDA could select useful wavenumber intervals to build the most simple and exact classification model. It is a feasible and promising method for quality-control and discrimination analysis of Chinese medicine.

\section{Theory}

Principle of moving window partial least-squares discriminant analysis (MWPLSDA) and construction of model

The principle of moving window partial least-squares discriminant analysis is that constructing a suitable window moves along the full spectral interval. The window starts at the $i$ th spectral channel, and ends as the $(i+H-1)$ th spectral channel. The starting position of the window is denoted as the window position. The spectra obtained in the continuous moving spectral window is as a submatrix, $\mathrm{x}_{\mathrm{i}}(N \times H$ matrix $)$ containing the $i$ th to the $(i+H-1)$ th columns of the training matrix, $X_{n \times p}$. PLS models with different numbers of LVs can then be built to relate the spectra in the window to the classfication of the samples. It is given by

$$
\mathrm{y}_{\mathrm{i}}=\mathrm{x}_{\mathrm{i}} \mathrm{b}_{\mathrm{i}, \mathrm{k}}+\mathrm{e}_{\mathrm{i}, \mathrm{k}}
$$

where $b_{i, k}(H \times 1)$ is the regression coefficients vector estimated by using PLS with the $k$ PLS components, and $\mathrm{e}_{\mathrm{i}, \mathrm{k}}$ is a residue vector obtained with a $k$-component PLS model. At each position, the PLS classification models with varying PLS component number is built for training samples, and the sums of squared residues (SSR) are calculated with these PLS models and plotted against the windows. Spectral intervals with lower classification errors and less latent variables are then selected and combined to develop a PLSDA model. ${ }^{14,15}$ The model can be described as follows:

$$
Y_{n \times k}=X_{n \times p} B_{p \times k}
$$

Here the classification matrix, $Y_{n \times k}$, contains the classification vectors of the training sets; each row is the classification vector of a sample. $n$ is the number of samples, $p$ is the number of wavelengths selected and $k$ is the number of classes. Herein, the classification vector $\mathrm{fj}$ (the $j$ th element is 1 and other elements are 0s) is used to code samples in group j. Each column of $Y_{n \times k}$ is regressed to spectral training matrix $X_{n \times p}$ by PLS1, and the number of the latent variables is selected by cross-validation. Each column of the regression matrix $B_{p \times k}$ is the regression coefficients vector of PLS1.

For unknown samples, the classification matrix $Y_{\text {un }}$ can be predicted by spectral matrix $X_{u n}$ of the unknown samples and regression matrix $B_{p \times k}$,

$$
Y_{u n}=X_{u n} B_{p \times k}
$$

which classes the unknown samples belong to can be determined according to the classification vectors of the classification matrix, $\mathrm{Y}_{\text {un }}$. The $i$ th unknown sample is ascribed to the $j$ th group when the maximal element in its classification vector appears at the $j$ th of position, which can be represented as:

$$
\mathrm{G}_{\mathrm{j}}=\operatorname{Index}_{\max }\left(\mathrm{Y}_{\text {uni... }}\right)
$$

where $G_{j}$ denotes the classification of the $i$ th sample, and Index $_{\max }$ indicates the position where the maximal element of a vector appears.

\section{Experimental}

\section{Apparatus and reagents}

The NIR spectra for Liuwei Dihuang pills were collected from an FT-IR/NIR spectrometer (Nexus 870, Thermo Electron Company, Madison, USA) equipped with an updrift sampling device (Thermo Electron Company) and an indium gallium arsenide (InGaAs) detector. Liuwei Dihuang samples were provided by five different manufactures as follows: Hiuren Corporation in Jiangxi, Jiuzhitang Corporation in Changsha, Taiji Corporation in Zhejiang, Tongrentang Corporation in Beijing, Wanxi Corporation in Henan.

\section{Acquisition of NIR data}

The NIR spectra were taken in the range of $10000-4000 \mathrm{~cm}^{-1}$ at a resolution of $8 \mathrm{~cm}^{-1}$. Sample scanning was acquired by diffuse reflectance. A total of 30 scans were accumulated per measurement. The spectra of thin glass slices as blank were collected before putting Liuwei Dihuang samples; 30 samples were selected randomly for every different corporation and the NIR spectra data were recorded. The samples of the five different corporations were respectively marked as follows: (001 - 030) denoting Hiuren Corporation, (031 - 060) Jiuzhitang Corporation, (061-090) Taiji Corporation, (091-120) Tongrentang Corporation, (121 - 150) Wanxi Corporation. 


\section{Pretreatment of the spectra}

The original NIR spectra of 150 samples of five kinds of Liuwei Dihuang were pre-processed by multiple scatter correct (MSC), ${ }^{16}$ followed by a second-derivative treatment.

\section{Data processing}

PCA, LDA, PLSDA, MWPLSDA programs were written in our laboratory and run on a MatLab environment (MathWorks, Natick, MA). Data were mean centered prior to building a training model. The 150 spectral data were randomly split into two groups; 101 spectra were used for training sets (Hiuren Corporation, 19; Jiuzhitang Corporation, 22; Taiji Corporation, 20; Tongrentang Corporation, 19; Wanxi Corporation, 21) and 49 spectra are used for prediction sets (Hiuren Corporation, 11; Jiuzhitang Corporation, 8; Taiji Corporation, 10; Tongrentang Corporation, 11; Wanxi Corporation, 9).

\section{Results and Discussion}

Comparison of different recognition methods for identification Principal-components analysis (PCA) ${ }^{17,18}$ is a multidimensional modelling method, which is based on the calculating of linear combinations between the variables, and explaining the greatest variance of the data. That is, the method enables a transformation of numerous original variables into new ones, called principal components, being linear combinations of source data. In this way the total number of variables can be reduced, because usually 2 or 3 principal components are sufficient to explain the majority of the total variability of the original magnitudes; PCA as a tool for information extracting has been used in near-infrared spectral application. In our work, the dimensionality of a Liuwei Dihuang dataset including a spectral matrix of 101 training samples and 49 prediction samples were reduced by PCA. Firstly, the projection of the original space was transformed into a two-way visual subspace. In this way, a principal component 1 (PC1) with the largest variance could be generated. Then, generating another principal component was orthogonal to PC1 as the second principal component (PC2); it represents the second axis of a larger residual variance. Once the first two PCs had been elucidated, their projection could be obtained in terms of the two sets of loadings. The scores of the first and the second principle components of the train and prediction sets as projection axis are plotted and are showed in Figs. 1(a) and 1(b), respectively. It can be seen that the five kinds of Liuwei Dihuang samples are hard to distinguish due to severe overlapping. Though it is effective for feature extraction and dimensionality reduction, some useful discriminate information was missing while reducing the dimension of data. Thus, PCA could not accurately discriminate the five kinds of Liuwei Dihuang samples.

Linear discriminant analysis (LDA) ${ }^{19,20}$ is a well-known technique for dimensionality reduction and feature extraction. The basic principle of LDA is to seek a set of orthogonal latent variables to represent the original feature space. The latent variables have the maximum between-group variance compared with the within-group variance of all the groups. The essence of this method is to extract a low-dimensional subspace with strong discriminant ability from the original feature space; then the scores of the first 2 or 3 latent variables are plotted. A spectral matrix of Liuwei Dihuang pills including 101 training samples and 49 prediction samples was decomposed to obtain the two main eigenvalues; the scores of the first two latent variables of the train and prediction sets are shown in Figs. 2(a) and 2(b). It can be seen that a training set of five kinds of Liuwei Dihuang
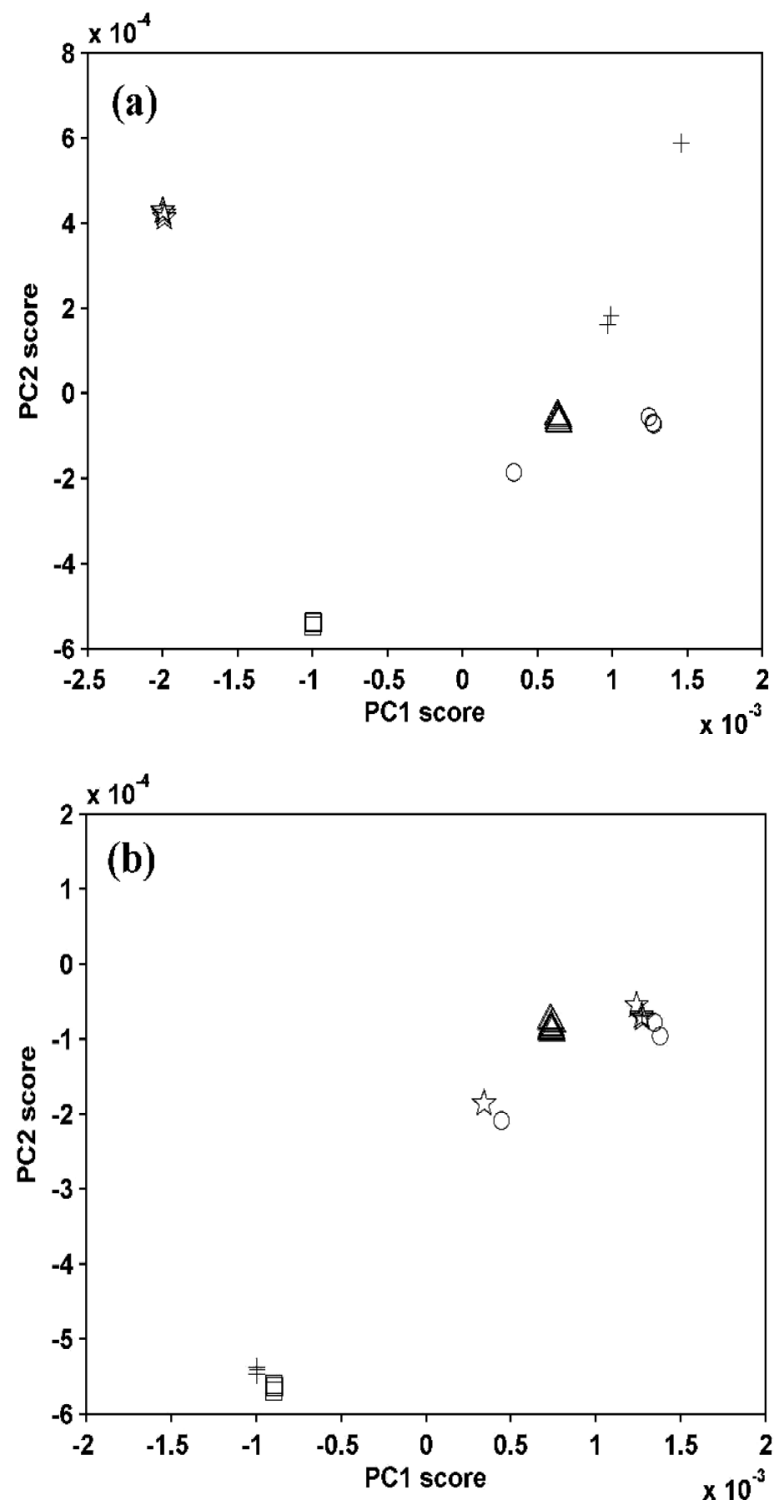

Fig. 1 Score plot for first and second principal components for the samples. (a) 101 training samples and (b) 49 prediction samples. $\triangle$, 19 training and 11 prediction samples from Huiren; $\bigcirc, 22$ training and 8 prediction samples from Jiuzhitang; $\square, 20$ training and 10 prediction samples from Taiji; is $^{2} 19$ training and 11 prediction samples from Tongrentang; +, 21 training and 9 prediction samples from Wanxi.

pills was identified accurately, but classification of the prediction sets suffered from overlapping and confusion. This implies that models built on training samples cannot be used to discriminate prediction samples. The result indicates that LDA has a good learning performance, but it would be less effective to classify unknown samples if the number of variables of the data matrix is much higher than the number of samples. There persist in LDA some limitations; it is the requirement that at least one the scatter matrices be nonsingular, and does not guarantee one to find the optimal subspace when the so-called "outlier class" is dominant in estimating the scatter matrices.

Partial least-squares discriminant analysis (PLSDA) ${ }^{21,22}$ is a linear-regression method based on all spectra information. The multivariate variables corresponding to the observations (spectral descriptors) are related to the class membership for each sample. PLSDA using the whole spectra region was used to relate the 

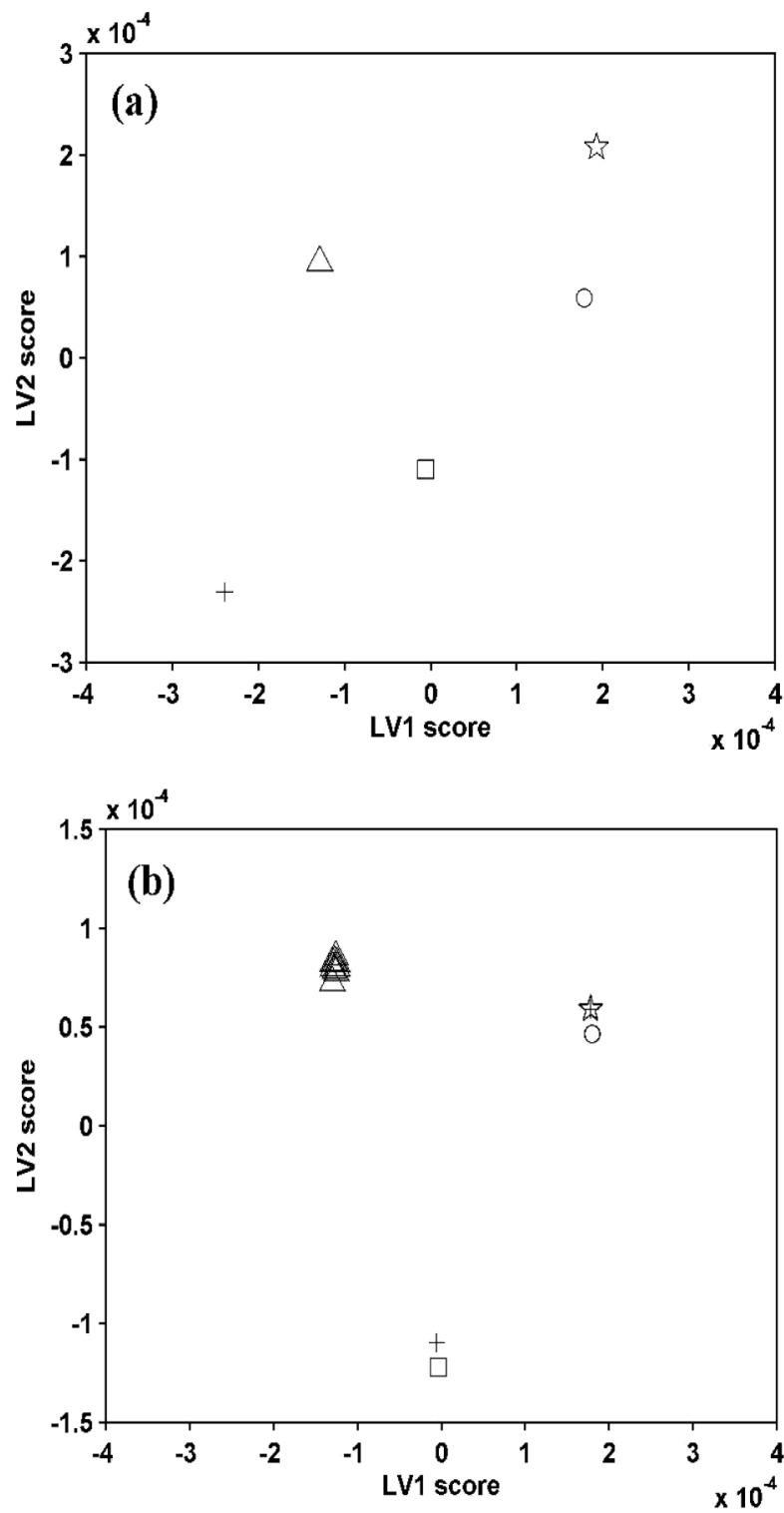

Fig. 2 Score plot for the first and second latent variable for the samples. (a) 101 training samples and (b) 49 prediction samples. Symbols are the same as those in Fig. 1.

dummy group codes to the full spectral variables, where the dummy codes for the five groups of samples were $(1,0,0,0,0)$, $(0,1,0,0,0),(0,0,1,0,0),(0,0,0,1,0)$ and $(0,0,0,0,1)$. A key problem must be determined. That is the optimum number of latent variables. A model built with too few variables is rarely sufficient and too many latent variables capture not only the information related to the discrimination problem, but also the noise and particularities of the calibration data. For the Liuwei Dihuang data, the PLSDA model based on full spectra, with the optimum number of six latent variables, was determined by six-fold cross-validation. The training set is split into six subsets, five with 17 samples each and one with 16 samples. Figures 3(a) and 3(b) show that plots of dummy codes of the training sets and prediction sets. Five kinds of Liuwei Dihuang samples were classified by position of the maximal dummy codes of the samples, respectively. The dummy code $\mathrm{f} 1(1,0$, $0,0,0)$ represent 19 training and 11 prediction samples from (001 - 030) samples, f2 $(0,1,0,0,0)$ represent 22 training and
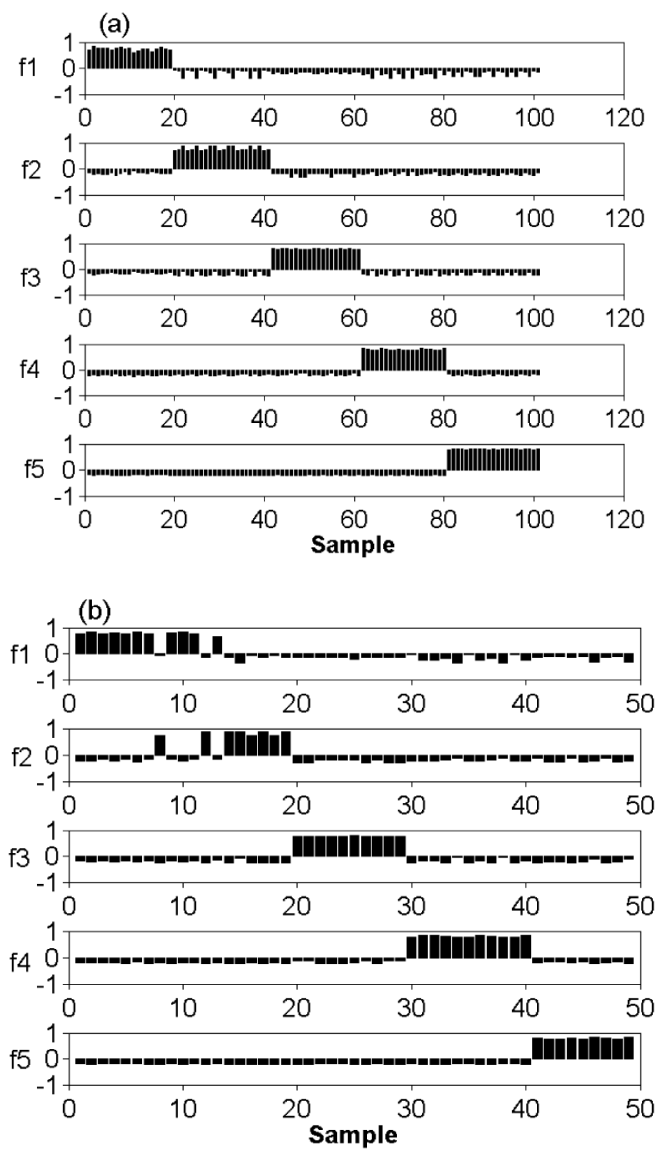

Fig. 3 Dummy vectors (f1, $\mathrm{f} 2, \mathrm{f} 3, \mathrm{f} 4, \mathrm{f} 5$ ) of PLSDA for the samples. (a) 101 training samples and (b) 51 prediction samples.

8 prediction samples from $(031-060)$ samples, f3 $(0,0,1,0,0)$ represent 20 training and 10 prediction samples from $(061-090)$ samples, $\mathrm{f} 4(0,0,0,1,0)$ represent 19 training and 11 prediction samples from (091 - 120) samples, and f5 $(0,0,0,0,1)$ represent 21 training and 9 prediction samples from $(121-150)$ samples. One can see that five kinds of Liuwei Dihuang samples in the training sets are identified accurately. However, two samples in the prediction sets were classified incorrectly. The 8th Liuwei Dihuang sample belonging to the f1 group was classified incorrectly into the $\mathrm{f} 2$ group. The 13th belonging to the f2 group was classified incorrectly into the f1 group. Perhaps the main cause is due to the fact that the prediction ability is interfered by some spectral variables in the whole spectral range containing uninformative data. PLS might still be susceptible to overfitting by introducing noninformative variables, limiting the practical usefulness of PLS in NIR spectroscopic classification problems. Usually, a PLSDA model established in a full spectral range suffers from possibly two disadvantages. Firstly, it will be time-consuming when the training data set is very large, especially for data sets containing thousands of wavenumbers. Moreover, the classification accuracy might be poor owing to interference of useless information and overlapping NIR bands, thereby limiting the practical usefulness of PLS in NIR spectroscopic classification problems.

Informative regions of the original NIR spectra and second derivative spectra of 150 samples of five kinds of Liuwei Duahuang pills as shown in Figs. 4(a) and 4(b) are discussed before using our strategy. One can see the spectra information region of five kinds of Liuwei Duahuang between 

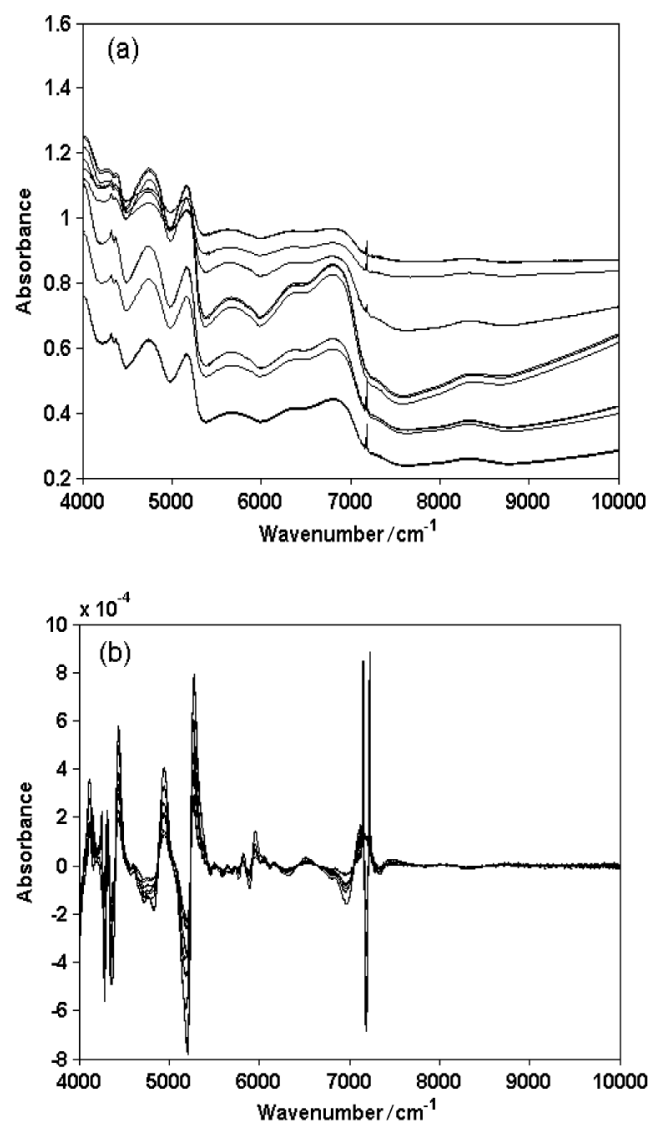

Fig. 4 NIR spectra of 150 samples of five kinds of Liuwei Duahuang pills. (a) Original spectra and (b) second-derivative spectra.

$4000-8800 \mathrm{~cm}^{-1}$, but the spectral region could contain lots of non-class-related and other adverse information. In addition, there are many crossovers and overlappings among these samples in original NIR spectra, which means the spectra of the five kinds of samples, are very similar. After a second-derivative treatment, a null derivative signal was observed in the regions with baseline shifts. The resolution was significantly improved accordingly. It is also noticeable that the second-derivative preprocessing, despite removing the baseline, may also enhance the noise in some spectral intervals. Therefore, it is necessary to eliminate intelligently interferential uninformative intervals by an effective strategy, and to construct an efficacious pattern-recognition model for discrimination of the samples varieties. For the sake of constructing of an efficacious model, a moving window partial least-squares discriminant analysis (MWPLSDA) is employed for achieving a satisfactory identification rate. Herein, the size of the window is chosen as 40 to obtain spectral intervals containing useful information. Residue lines obtained by MWPLS for training sets are shown in Fig. 5. Note that the right boundary of the spectral region can be extended by the size of the window minus one, 39. It can be seen that when the window was located in the spectral intervals of $5025-5539(5500+39) \mathrm{cm}^{-1}, 6920-7259(7220+39) \mathrm{cm}^{-1}$ and $7850-8089(8050+39) \mathrm{cm}^{-1}$, the PLS models could achieve the least complexity and low SSR. Thus, the spectral intervals of $5025-5539,6920-7259$ and $7850-8089 \mathrm{~cm}^{-1}$ are selected and combined to develop a PLSDA model for the identification of different kinds of Liuwei Dihuang samples. The PLSDA with a moving window technology is used to relate

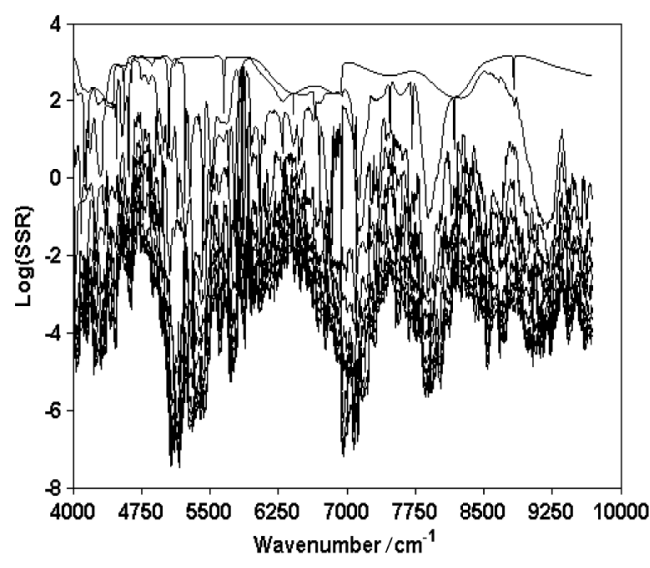

Fig. 5 Residue line obtained by MWPLS for the training sets.
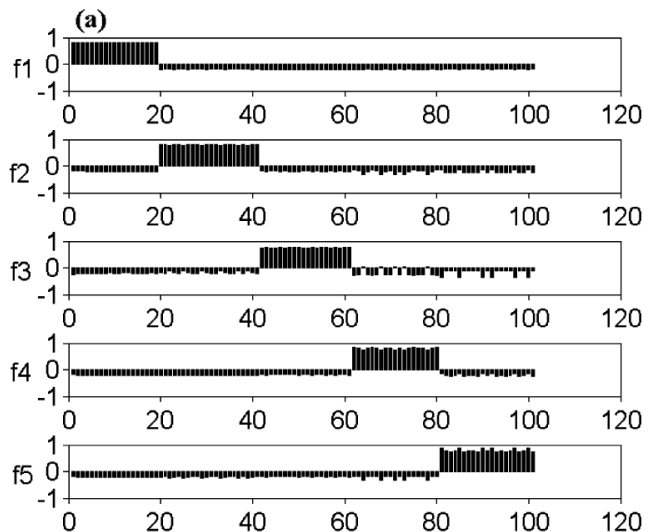

(b)

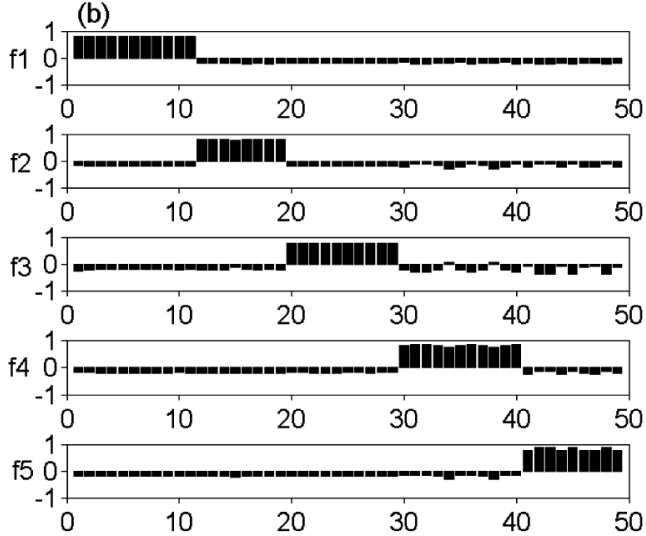

Fig. 6 Dummy vectors (f1, f2, f3, f4, f5) of MWPLSDA for the samples. (a) 101 training samples and (b) 51 prediction samples.

the dummy group codes to the selected spectral variables, where the dummy codes for the five groups of samples are the same with PLSDA without moving window technology. The optimum number of the model is determined as six latent variables by aforementioned six-fold cross-validation. Figures 6(a) and 6(b) show that plots of the dummy codes of the training sets and the prediction sets in the MWPLSDA model. The dummy codes $\mathrm{f} 1$, $\mathrm{f} 2, \mathrm{f} 3, \mathrm{f} 4$ and $\mathrm{f} 5$ respesent training and prediction samples from each variety of samples, are also the same as aforementioned that of PLSDA model based on the whole spectral region. Five kinds of Liuwei Dihuang samples are also classified by position 
of the maximal dummy codes of samples, respectively. One can see that five kinds of Liuwei Dihuang samples in the training sets were identified accurately, and in the prediction sets were also accurately classified. The total accuracy rates are $100 \%$. Uninformative and interferential regions for classification can be removed, and the prediction ability of model is improved compared with the aforementioned pattern-recognition methods At the same time, the points of wavenumber variables are also significantly reduced. Accordingly, it could achieve good recognition, much more quickly.

\section{Conclusion}

A comparison among PCA, LDA, PLSDA and MWPLSDA methods for the identification of traditional Chinese medicine Liuwei Dihuang pills by NIR was carried out. The results discussed above demonstrate that MWPLSDA is proposed to be superior to the other three conventional pattern-recognition methods. The MWPLSDA method could select narrow, but informative wavelength intervals to reconstruct an efficacious classification model, which gives high identification accuracy and a very low storage requirement by removing uninformative wavelength intervals, and then fast recognition and excellent data prediction could be obtained. Hence, the identification method is a feasible and promising method for the quality control and discrimination of traditional Chinese medicine.

\section{Acknowledgements}

The work was financially supported by the National Natural Science Foundation of China (Grant Nos. 20605007, 20775025) and "973" National Key Basic Research Program of China (2007CB216404, 2007CB310500).

\section{References}

1. The Pharmacopoeia Committee of PRC, Pharmacopoeia of the People's Republic of China Part 1, 2000, Chemical Industry Press, Beijing, 415.
2. J. X. Zou, Mod. Diagn. Treat, 2004, 15, 174.

3. Y. A. Woo, H. J. Kim, K. R. Ze, and H. Chung, J. Pharm. Biomed. Anal., 2005, 36, 955.

4. M. Laasonen, T. Harmia-Pulkkinen, C. L. Simard, E. Michiels, M. Rasanen, and H. Vuorela, Anal. Chem., 2002, 74, 2493.

5. H. Y. Fu, S. Y. Huan, L. Xu, L. J. Tang, J. H. Jiang, H. L.Wu, G. L. Shen, and R. Q. Yu, J. Near Infrared Spectrosc., 2007, 15, 291.

6. J. H. Jiang, R. J. Berry, H. W. Siesler, and Y. Ozaki, Anal. Chem., 2002, 74, 3555.

7. S. Kasemsumran, Y. P. Du, K. Maruo, and Y. Ozaki, Anal. Chim. Acta, 2004, 526, 193.

8. N. Kang, S. Kasemsumran, Y. A. Woo, H. J. Kim, and Y. Ozaki, Chemom. Intell. Lab. Syst., 2006, 82, 90.

9. S. Kasemsumran, Y. P. Du, K. Murayama, M. Huehne, and Y. Ozaki, Anal. Chim. Acta, 2004, 512, 223.

10. S. Kasemsumran, Y. P. Du, K. Maruo, and Y. Ozaki, Chemom. Intell. Lab. Syst., 2006, 82, 97.

11. Y. P. Du, Y. Z. Liang, J. H. Jiang, R. J. Berry, and Y. Ozaki, Anal. Chim. Acta, 2004, 501, 183.

12. Y. Lee, H. Chung, and A. Arnold, Anal. Chim. Acta, 2006, 572,93

13. S. Sasic, Y. Katsumoto, H. Sato, and Y. Ozaki, Anal. Chem., 2003, 75, 4010.

14. B. K. Alsberg, D. B. Kell, and R. Goodacre, Anal. Chem., 1998, 70, 4126.

15. U. Lutz, R. W. Lutz, and W. K. Lutz, Anal. Chem., 2006, $78,4564$.

16. H. Martens, J. P. Nielsen, and S. B. Engelsen, Anal. Chem., 2003, 75, 94

17. E. K. Keemley, Chemom. Intell. Lab. Syst., 1996, 33, 47.

18. S. Alok, K. P. Kuldip, and C. O. Godfrey, Pattern Recogn., 2006, 7, 1215

19. G. A. Bakken and P. C. Jurs, J. Med. Chem., 2000, 43, 4534.

20. J. Yang and J. Y. Yang, Pattern Recogn., 2003, 36, 56.

21. B. K. Alsberg, D. B. Kell, and R. Goodacre, Anal. Chem., 1998, 70, 4126.

22. U. Lutz, R. W. Lutz, and W. K. Lutz, Anal. Chem., 2006 , $78,4564$. 